\section{Biosimilars: lights and shadows in rheumatology}

\author{
Monica Todoerti, Roberto Caporali, \\ Francesca De Nard, Nicola Boffini, \\ Garifallia Sakellariou, Maria Eva Romano, \\ Lorenzo Cavagna \\ Division of Rheumatology, IRCCS \\ Foundation Policlinic S. Matteo, Pavia, \\ Italy
}

\section{Abstract}

In the last 10 years, the growing approval and marketing of biological agents has significantly ameliorated the outcomes of rheumatoid arthritis and spondyloarthritis patients suffering from active and refractory disease despite conventional treatments. As patent protection of many biopharmaceuticals will expire in the next years, biosimilars could be proximally introduced. Such agents could be marked only when they will be proven, through in vitro and in vivo studies, to be similar enough to the original comparator in term of quality, efficacy and safety. As biosimilars are less expensive than corresponding originators, a wider use of these drugs may substantially cut off the expenditure of biopharmaceuticals. Nevertheless, ongoing debate exists in scientific community: the intrinsic complex and large structure of biologic molecules besides the natural variability in the manufacturing processes might lead to a slightly different product respect to the original one, so that relevant implications for efficacy and safety concerns might arise, especially in the long-term period. Immunogenicity and extended indications of biosimilars represent further matter of discussion, too. Thus, before their approval and marketing, specific guidelines and steps imposed by national and/or international regulatory agencies should be followed along with the respect of scientific societies position in each specific contest.

\section{Introduction}

The recent advent of biosimilars represents the most innovative aspect in the treatment of rheumatoid arthritis (RA) with biological disease modifying anti-rheumatic drugs (bDMARDs). As recently introduced by Smolen et al., ${ }^{1}$ such innovation might require, as a consequence, the proposal of introducing a new nomenclature for all the available DMARDs for RA patients: synthetic (or chemical) DMARDs, divided into conventional syn- thetic (csDMARDs: like methotrexate, and all the other methotrexate-equivalent DMARDs) and targeted synthetic (tsDMARDs, such as tofacitinib, fostamatinib, baricitinib or apremilast, or agents not focused primarily on rheumatic diseases, such as imatinib or ibrutinib respectively), and biologic DMARDs, including biological original and biosimilars (boDMARDs and bsDMARDs, respectively). ${ }^{1}$

Such proposed nomenclature covers not only the simple nosological issues, in order to specify the clear nature and identity of each compound, but it also indirectly arises several problems like the complexity of approval process by authorities, drug production and marketing. This new recognized drug classification also represents the starting point to allow a better comparison among such different classes of agents.

\section{Study design}

A revision of the most relevant issues related to biosimilars in rheumatology has been assessed. In the following sections several topics will be briefly discussed in accordance with the available knowledge in the field.

\section{Biosimilars: definition}

Biosimilars are biological agents that essentially copy the primary, secondary and tertiary structures of a parent originator compound, ideally even including its post-translational modifications; in addition, they should convey similar efficacy and safety as the original protein. ${ }^{2}$ The potential availability of biosimilar versions of these targeted drugs might promise larger accessibility to biopharmaceuticals with similar efficacy and safety but at a lower cost, possibly marketed whenever original branded have expired. These second-generation products could not be merely considered as identical follow-on products, in comparison to the first generation ones, as it happens for simpler chemical drugs. In fact, while for chemical compounds a generic drug is intended to contain the same active component, normally a small molecule, which is qualitatively and quantitatively identical to the reference drug, although the excipients may differ, for biological compounds, like anti-TNF agents, biosimilars might not be fully identical to the original comparator. The greater dimensions together with the more complexity and spontaneous and/or induced variability of such biologic molecules, partly dependent on spontaneous/ forced minor and major manufacturing modifications, should explain the great challenge of biosimilars. Specifically, many factors should
Correspondence: Monica Todoerti, Division of Rheumatology, University of Pavia and IRCCS Foundation Policlinic S. Matteo, viale Golgi 2, 27100 Pavia, Italy.

Tel.: +39.0382.501.878 - Fax: +39.0382.503.171.

E-mail: monica.todoerti@libero.it

Key words: biosimilars, rheumatoid arthritis, spondyloarthropathies.

Contributions: MT reviewed main evidences on the topic in available current scientific literature and was responsible for the design of the manuscript; LC was mainly involved in the original conception of the work. All the authors equally contributed to revise scientific evidences.

Conflict of interests: the authors declare no potential conflict of interests.

Received for publication: 13 June 2014.

Revision received: 28 August 2014.

Accepted for publication: 1 September 2014.

This work is licensed under a Creative Commons Attribution NonCommercial 3.0 License (CC BYNC 3.0).

@C Copyright M. Todoerti et al., 2014

Licensee PAGEPress, Italy

Rheumatology Reports 2014; 6:5518

doi:10.4081/rr.2014.5518

be taken into account in the field: i) a large biologic, such as a monoclonal antibody, measures about $150 \mathrm{Kda}$ and has more than a 1000 aminoacids; ii) it usually degrades over time, requiring special storage to maintain stability; iii) expression systems should be stable too; iv) manufacturing changes are not rare, sometimes even directly done by the original developers of biologics in order to find additional beneficial effects; v) in addition, some specific post-translational modifications may also be critical for function. As a consequence, while marketing the generic form of simple chemical compounds requires only the preventive demonstration of its bioequivalence respect to the reference drug, that is normally done in healthy volunteers to compare the bioavailability between the tested and the reference product, marketing a biosimilar requires a more complex and longer multi-step process which not always achieves success (Table 1). Taking this into account, we can wonder whether biosimilars could really result similar enough to the original products. This could explain the existence of a great regulatory framework as a fundamental pre-requisite to admit their final approval and marketing: pre-clinical and clinical studies are preliminary required with important implications. In fact, biosimilars should be intended to be sufficiently similar to 
the reference products, that there is no clinically meaningful difference between them in terms of purity, safety, and efficacy. The same therapeutic effect could be thus obtained with a cheaper version of the drug, which could be marketed only after the patent of a branded agent has definitively expired.

\section{Biosimilars and pharmaco-economical issues}

Although the world-life prevalence of RA and ankylosing spondylitis (AS) is not high, the chronic course of such diseases and their irreversible consequences in term of damage and disability affecting relatively young and working patients, have great impact on social and economical panorama. Direct and indirect costs related to patients and disease themselves are high, and they have been progressively implemented by drugs costs, especially after the introduction of biological agents. One can wonder whether such implemented drugs cost might be counterbalanced by saving other resources or by avoiding other health care costs through better patients outcomes, especially with functional and work ability preservation. Cost-effectiveness analyses should be optimized in order to quantify the effect of such new treatments, like bDMARDs, in chronic diseases, but concerns exist due to the fact that clinical trials are generally short compared with the duration of the disease itself, and limited pharmaco-economical data on the use of this new treatments are available in clinical practice..$^{3,4}$ In this contest, a disease model should be created as a baseline paradigm to compare cost-effectiveness of new treatments in RA: it must comprehend epidemiologic data related to the disease itself, information about resource consumption at any disease severity level, entity of direct and indirect costs related to patients morbidity and disability. The evidences so far available in this direction seem to support and admit advantages of TNF antagonists, compared with traditional therapies for RA; biosimilars could be of further additional value for such pharmaco-economical purposes: similar efficacy and safety profiles could go ahead with greater cost-saving prospects and, as a consequence, larger access for patients.

\section{Biosimilars and regulatory concerns}

All the regulatory agencies, like the Food and Drug Administration (FDA) and the European Medicines Agency (EMA) necessarily require a multi-step process through which in vitro and in vivo experiments could allow equivalence demonstration among original and biosimilars, relying on precise and specific preliminary definitions, which should be finally satisfied.

For the EMA, a biosimilar is a [...] biological medicinal product that contains a version of the active substance of an already authorized original biological medicinal product (reference medicinal product). A biosimilar demonstrates similarity to the reference medicinal product in terms of quality characteristics, biological activity, safety and efficacy based on a comprehensive comparability exercise.

According to the FDA, a biosimilar could be intended when [...] the biological product is highly similar to the reference product notwithstanding minor differences in clinically inactive components. The FDA specifically requires that clinical studies must show that there are no clinically meaningful differences between the biosimilar and the reference biopharmaceutical in the safety, purity, and potency of the product, while the EMA requires an appropriate comparability exercise in order to demonstrate that the similar biological and reference medicinal products have similar profiles in terms of quality, safety and efficacy.5.6 These studies must demonstrate that pharmacokinetics and pharmacodynamics, efficacy, and safety of the biosimilar are essentially equivalent to those of the innovator biopharmaceutical at the same dose, and that the biosimilar is not more immunogenic than the reference product. Because the therapeutic doses of the innovator biopharmaceutical have already been established and the biosimilar must be administered at the same doses, the development program for a biosimilar does not need to include dose-ranging studies in patients, like for the original product. Instead, at least one non-inferiority clinical trial comparing the biosimilar and the innovator reference biopharmaceutical is required to show that there are no significant differences in efficacy between the two drugs, and indeed that a biosimilar is non-inferior to the original compound in term of frequency and timing of achievement of predefined responses.

CT-P13 is a biosimilar infliximab that was compared to innovator infliximab both with invitro analytical studies, which demonstrated similar biologic effects (tumor necrosis factor /TNF neutralizing potency, and complementdependent cytotoxicity activity) and with in vivo studies, which showed similar clinical profiles. Thus, CT-P13 was studied in two randomized, double-blind, parallel-group, prospective clinical trials to assess its potential noninferiority to the reference product with respect to efficacy and safety outcomes over 30 weeks. The PLANETAS trial was a phase 1 study that compared the pharmacokinetics, efficacy, and safety of CT-P13 to those of innovator infliximab, each as monotherapy in patients with $\mathrm{AS}^{7}$ The PLANETAS trial achieved its primary and secondary endpoints, demonstrating that pharmacokinetic profiles of CT-P13 and innovator infliximab were equivalent between weeks 22 and 30 , and highly similar between treatment groups for each of the six intravenous doses that were infused over the 30-week study. The PLANETRA trial was a phase 3 study that evaluated the efficacy and safety of CT-P13 compared to innovator infliximab, each in combination with methotrexate, in patients with active RA. ${ }^{8}$ In both studies, a quite similar clinical efficacy

Table 1. Some of the available ongoing biosimilars and corresponding results of approving process.

\begin{tabular}{ll} 
Product & Results \\
Interferon $\alpha 2 \mathrm{~A}$ & No approval \\
Interferon $\beta 1 \mathrm{~A}$ : avonex & Initial production stopped; subsequent product improved \\
\hline rHuEPO: eprex & Aggregate formation led to development of anti-EPO antibodies and 175 cases of pure red cell aplasia happened in 1998-2004 \\
p55TNF-R:Ig: lenercept & Development discontinued \\
\hline Darbopoetin $\alpha$ : aranesp & Iterative manufacturing change \\
Rituximab: mabthera & Iterative manufacturing change \\
\hline p75TNF-R:Ig: etanercept: enbrel & Iterative manufacturing change \\
\hline
\end{tabular}

Modified from Dorner et al. ${ }^{2}$ 
was demonstrated in clinical end-points in either RA and AS patients in term of ACR and EULAR responses and ASAS criteria, respectively. Time of response was similar in both patients' populations too, with early effect maintained up to 30 and 54 weeks, as recently presented at EULAR Congress in Madrid in 2013: rapidity and sustainability have been finally demonstrated to be similar for both compounds. . $^{7,8}$

\section{Biosimilars and extension of indications}

After initial approval, FDA and EMA also advocate the introduction of biosimilars for additional and different indications, by extrapolation from the reference drug results and from data of biosimilar in the primary indication, without requiring substantial clinical and specific data: this process is called indication extrapolation and it might allow the extension of indication for the biosimilar itself. ${ }^{2}$ For instance, in 2013 the EMA recommended the marketing authorization of CT-P13, the world's first follow-on monoclonal antibody claimed to be biosimilar to infliximab (Remicade $®$, Janssen Biotech Inc., Horsham, PA, USA). ${ }^{9}$ The approval or recommended approval of CT-P13 was based on a single equivalence trial conducted in patients with RA, supplemented by a pharmacokinetic study on AS. 7,8 What was unique about this approval was that four other indications of infliximab (i.e., Crohn's disease, ulcerative colitis, psoriatic arthritis, and plaque psoriasis) were granted to CT-P13, although the product was not tested in these indicated populations. Such issue raised several concerns due to the observations that not all the extrapolation requirements by the regulatory agencies have been largely satisfied by the existing scientific and clinical data. RA itself might be not a sensitive model for all the other indications; moreover, clinically relevant mechanisms of action for infliximab, and thereby for CT-P13, could differ across the approved indications. If the biosimilar and the innovator biopharmaceutical share the same mechanisms of action in the various disease states and the requirements for licensure as a biosimilar have been met, the biosimilar might be licensed for use in some or all of the other indications for which the innovator biopharmaceutical is licensed. Although, careful monitoring is mandatory especially for such indications not directly explored.

Specific extrapolation criteria have been developed by regulatory agencies in order to mitigate concerns. Extended indications should be properly indicated in the product label: where the mood of action of investigated drugs varies across different indications or they are not fully understood, separated trials should be performed before extrapolation; individual patients and/or disease characteristics could influence results in efficacy and safety so that careful consideration must be given to such elements of variability. ${ }^{2}$ It remains to be seen whether physicians will be comfortable with prescribing in clinical practice a medication for an extended indication without supporting full phase III data.

\section{Biosimilars and immunogenicity}

The regulatory agencies absolutely require that the immunogenicity profile of follow-on products should be sufficiently characterized before extrapolation to other indications is sought. This might be taken into account, considering that the proportion of patients developing immunogenicity after treatment with infliximab varied widely by indication: it is higher in Crohn's disease (up to 61\%), ranging between 20 and $51 \%$ in psoriasis, lower in RA patients, probably partly due to the licensed indication of co-administration with methotrexate. ${ }^{10-12}$ This further explains why extrapolation of the results on the immunogenicity profile of CT-P13 could be inappropriate. In PLANETRA and PLANETAS trials, both CT-P13 and innovator infliximab were immunogenic, and levels of anti-drug antibodies were similar for both biopharmaceuticals. Specifically, antibodies to infliximab were detected up to a third of AS patients for each group (at week 24 in $27.4 \%$ and $22.5 \%$ for CT-P13 or innovator infliximab as monotherapy, respectively), and in nearly half of RA patients treated with either CT-P13 or innovator infliximab, each in combination with methotrexate. In both of these clinical trials, the proportion of clinical responders was lower among those with anti-drug antibodies than among those without. In addition, it has been recently reported that a rituximab biosimilar has produced anaphylactic reactions after the patients were switched to the biosimilar in Mexico and this raises the real issue of what should be the real definition of the biosimilarity and of the bioequivalence in terms of safety and immunogenicity. ${ }^{13}$ For the EMA agency, specific end-points should be addressed for the full characterization of the immunogenicity profile of biosimilars: characteristics of the drug itself and its short and long-term exposure effects, but also of the underlying disease, should be entirely considered. Therefore, immunogenicity of non endogenous proteins arises several concerns for safety and efficacy issues: as a direct corollary to this, biosimilars should have their own specific name and traceability both for epidemiological purposes, like in registries, and for spontaneous reporting/healthcare databases, too.

\section{Biosimilars: the present}

All biosimilars, intended globally as a group, now labeled by regulatory agencies enter into 3 categories: i) growth hormones, ii) erythropoietin, iii) granulocyte colony stimulating factors. $^{14}$ In the field of rheumatology, currently, several products labeled as biosimilars are approved for treatment of RA in a number of countries that, at the time of approval, did not have stringent regulatory processes in place to ensure comparability as defined by EMA and FDA and had stringent economic pressure. For these reasons, they could not be entirely considered as real biosimilars, but like intended copies. $^{2}$ On the contrary, currently, there are no biosimilars definitively approved by EMA or FDA for treatment of rheumatic diseases in Europe and in the USA: several randomized controlled trials (RCTs) are complete or on going in the view to attain a biosimilar status for treatment of rheumatic diseases. ${ }^{2}$ Earlier in 2012, the South Korean company Celltrion, failed for EMA approval of a biosimilar infliximab product, CTP13, based on results from the 2 previously presented large RCTs (PLANETRA and PLANETAS studies). In the recent 2013 update of recommendations for RA management, biosimilar issue has been briefly addressed too, but the role of such agents has not yet been fully specified. ${ }^{15}$

\section{Biosimilars: the future}

In the USA, the patent for innovator infliximab does not expire until September 2018, and thus biosimilar infliximab will not become available in the USA until after that date. ${ }^{16}$ Because the patent for innovator infliximab in the European Union does not expire until August 2014, CT-P13 will not be marketed in European Union countries until after that date. One financial analyst has estimated that, by 2018 , biosimilars will occupy a $40 \%$ share of the European market for therapeutic monoclonal antibodies. ${ }^{17}$ Specifically in the italian panorama, the actually shared position has been expressed in a defined paper: most biosimilars, whenever definitely approved, should be considered only for the indications already tested through comparability tests. Further extended indications, like sero-negative arthritis related to inflammatory bowel disease, off-label indications and/or in pediatric populations, should be rigorously explored with specific trials, attesting the equivalence in efficacy and safety standards towards the original comparator drug. 


\section{Biosimilars: considerations}

Considering all the issues discussed above, we can conclude with a concise list of relevant considerations on biosimilars: i) the complex structure and the great dimension of biological agents make difficult to produce really identical follow-on products, since even minor preventable and not-preventable modifications during the manufacturing process might considerably alter the drug structure and its profile; ii) biosimilars are not generic biologics and marketing approval for a biosimilar requires specific in vitro and in vivo studies which might support similarity among compounds; similarity should not be considered as a strict equivalence with the original comparator, but as an effort for this, without affecting the performance of the drug itself; iii) each approved biosimilar is considered to be a new product with all of the associated regulatory requirements; iv) comparative clinical trials are critically important to demonstrate the similarity in efficacy and safety profiles between the biosimilar and the innovator drug; this means that they have to be tested in humans, and that the testing has to be robust enough to detect any differences; v) whenever fully available for use in clinical practice, decisions to substitute one similar product with another should only be made at a physician's discretion and each biologic product must have a unique product name, so as to precisely identify and trace it; vi) as a consequence, interchangeability and exchange could not be applied to biosimilars, as it happens for simple chemical compounds: a product is considered as interchangeable, when it could substitute the originator without the authorization of the health care provider; on the contrary, up to now substitution of an originator with a biosimilar should be defined at a state level and following the opinion of a qualified health professional; vii) specific clinical trials should be conducted for other indications for which efficacy of the original compounds has been already admitted; viii) RA model can not be sufficient enough to directly extend indication from it to other different conditions; ix) in the pediatric field, further studies are absolutely mandatory before affirming equivalence; $x$ ) cost must not override safety and efficacy; the cost of biosimilars is clearly less than that of the brand name biologic to which it is similar, but this could not be the most relevant aspect to consider in the choice of the drug; xi) strict post-marketing surveillance must be followed: monitoring of biosimilars must conform to the same rigorous standards as those used for brand name biologics, with both real life registries and spontaneous reporting/healthcare databases; xii) their traceability must be assured through unique names. A registry, similar to, or integrated with those currently in use for original biological will be required to provide longer term monitoring of each specific, named, drug. Whether the required funding will be provided by the industry or other sources may vary.

\section{Conclusions}

In conclusion, in the immediate future the clinician's choice between the original and biosimilar compounds should be completely shared and discuss with the patients, in order to clearly offer them the most efficacious and safe treatments, and, if possible, at the cheapest cost; anyway, the main driver in this choice should always be the existence of sufficient evidence-based-medicine data along with a patient-first approach, that is in the first and main interest and benefit to the patients themselves. ${ }^{18}$ Thus, biosimilars might offer a good alternative to the existing biologic drugs only when complete and robust data might ensure their extensive use without safety and efficacy concerns for both tested and non tested (extended) indications.

\section{References}

1. Smolen JS, van der Heijde D, Machold KP, et al. Proposal for a new nomenclature of disease-modifying antirheumatic drugs. Ann Rheum Dis 2014;73:3-5.

2. Dorner T, Strand V, Castaneda-Hernandez $\mathrm{G}$, et al. The role of biosimilars in the treatment of rheumatic diseases. Ann Rheum Dis 2013;72:322-8.

3. Lapadula G, Ferraccioli GF. Biosimilars in rheumatology: pharmacological and pharmacoeconomic issues. Clin Exp Rheumatol 2012;30:S102-6.

4. Caporali R, Bobbio Pallavicini F, Filippini $\mathrm{M}$, et al. Treatment of rheumatoid arthritis with anti-TNF-alpha agents: a reappraisal. Autoimmun Rev 2009;8:274-80.

5. United States Department of Health and Human Services, Food and Drug Administration (US). H.R. 3590-686. Patient protection and affordable care act. Title VII - improving access to innovative medical therapies. Subtitle A Biologics price competition and innovation. Sec. 7002. Approval pathway for biosimilar biological products. 2010 . Available from: http://www.fda.gov/down loads/Drugs/GuidanceComplianceRegulat oryInformation/UCM216146.pdf. Accessed on: 6 May 2013.

6. European Medicine Agency. Guideline on similar biological medicinal products containing biotechnology-derived proteins as active substance: non-clinical and clinical issues. 2006. Available from: http://www. ema.europa.eu/docs/en_GB/document_lib rary/Scientific_guideline/2009/09/WC5000 03920.pdf. Accessed on: 6 May 2013.

7. Park W, Hrycaj P, Jeka S, et al. A randomised, double-blind, multicenter, parallel-group, prospective study comparing the pharmacokinetics, safety and efficacy of CT-P13 and innovator infliximab in patients with ankylosing spondylitis: the PLANETAS study. Ann Rheum Dis 2013;72: 1605-12.

8. Yoo DH, Hrycaj P, Miranda P, et al. A randomised, double-blind, parallel-group study to demonstrate equivalence in efficacy and safety of CT-P13 compared with innovator infliximab when co-administered with methotrexate in patients with active rheumatoid arthritis: the PLANETRA study. Ann Rheum Dis 2013;72:1613-20.

9. European Medicines Agency. European Medicines Agency recommends approval of first two monoclonal-antibody biosimilars. 2013. Available from: http:// www.ema. europa.eu/ema/index.jsp?curl=pages/news _and_events/news/2013/06/news_detail_0 01837.jsp\&mid=WC0b01 ac058004d5c1. Accessed on: 29 July 2013.

10. Menter A, Feldman SR, Weinstein GD, et al. A randomized comparison of continuous vs. intermittent infliximab maintenance regimens over 1 year in the treatment of moderate-to-severe plaque psoriasis. J Am Acad Dermatol 2007;56:e1-15.

11. Baert F, Noman M, Vermeire S, et al. Influence of immunogenicity on the longterm efficacy of infliximab in Crohn's disease. N Engl J Med 2003;348:601-8.

12. Flood J. Tumor necrosis factor inhibitors in the treatment of chronic inflammatory diseases. A review of immunogenicity and potential implications. Manag Care 2009; 18:1-5.

13. COFEPRIS. Comunicado a los profesionales de la salud. Reacciones anafilácticas por el uso de Rituximab. Available from: www. cofepris.gob.mx/AZ/Documents/Farmacovig ilancia/Comunicado\%20Rituximab.pdf

14. European Medicine Agency. Human medicines - Biosimilars. 201318 January 2013. Available from: http:/www.ema.europa.eu/ ema/index.jsp?curl=pages/regulation/general/general_content_000408.jsp\&mid=W C0b01ac058002958c

15. Smolen JS, Landewé R, Breedveld FC, et al. EULAR recommendations for the management of rheumatoid arthritis with synthetic and biological disease-modifying antirheumatic drugs: 2013 update. Ann Rheum Dis 2014;73:492-509.

16. GaBI Online. US $\$ 67$ billion worth of 
biosimilar patents expiring before 2020 . 2012. Available from: http://www.gabionline.net/Biosimilars/General/US-67-billion-worth-of-biosimilar-patents-expiringbefore-2020. Accessed on: 6 May 2013.
17. Jack A. Regulators pave way for biosimilar drugs. Financial Times 29-30 June 2013.

18. Lee E0, Emanuel EJ. Shared decision making to improve care and reduce costs. N Engl J Med 2013;368:6-8. 